\title{
TITLE:
}

\section{Asymmetric orientation of toluene molecules at oil-silica interfaces}

\section{$\operatorname{AUTHOR}(S)$ :}

Ledyastuti, Mia; Liang, Yunfeng; Kunieda, Makoto; Matsuoka, Toshifumi

\section{CITATION:}

Ledyastuti, Mia ...[et al]. Asymmetric orientation of toluene molecules at oil-silica interfaces. Journal of Chemical Physics 2012, 137(6): 064703.

\section{ISSUE DATE:}

2012-08-09

URL:

http://hdl.handle.net/2433/187979

\section{RIGHT:}

(c) 2012 American Institute of Physics. This article may be downloaded for personal use only. Any other use requires prior permission of the author and the American Institute of Physics. 


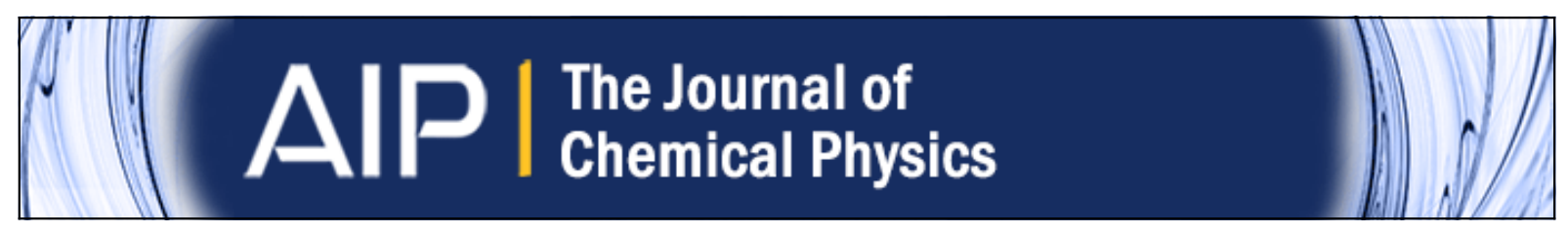

\section{Asymmetric orientation of toluene molecules at oil-silica interfaces}

Mia Ledyastuti, Yunfeng Liang, Makoto Kunieda, and Toshifumi Matsuoka

Citation: The Journal of Chemical Physics 137, 064703 (2012); doi: 10.1063/1.4742696

View online: http://dx.doi.org/10.1063/1.4742696

View Table of Contents: http://scitation.aip.org/content/aip/journal/jcp/137/6?ver=pdfcov

Published by the AIP Publishing

Articles you may be interested in

Structure and dynamics of TIP3P, TIP4P, and TIP5P water near smooth and atomistic walls of different hydroaffinity

J. Chem. Phys. 140, 174501 (2014); 10.1063/1.4872239

Dielectric spectra broadening as a signature for dipole-matrix interaction. IV. Water in amino acids solutions

J. Chem. Phys. 140, 135104 (2014); 10.1063/1.4869542

Hydrophobic and hydrophilic interactions in aqueous mixtures of alcohols at a hydrophobic surface

J. Chem. Phys. 139, 114706 (2013); 10.1063/1.4821604

Terahertz absorption of dilute aqueous solutions

J. Chem. Phys. 137, 235103 (2012); 10.1063/1.4772000

Structure and dynamics of liquid methanol confined within functionalized silica nanopores

J. Chem. Phys. 133, 154707 (2010); 10.1063/1.3503886

\section{AlP Re-register for Table of Content Alerts}




\title{
Asymmetric orientation of toluene molecules at oil-silica interfaces
}

\author{
Mia Ledyastuti, Yunfeng Liang, a) Makoto Kunieda, and Toshifumi Matsuoka ${ }^{\text {a) }}$ \\ Environment and Resource System Engineering, Kyoto University, Kyoto 615-8540, Japan
}

(Received 7 May 2012; accepted 23 July 2012; published online 9 August 2012)

\begin{abstract}
The interfacial structure of heptane and toluene at oil-silica interfaces has previously been studied by sum frequency generation [Z. Yang et al., J. Phys. Chem. C. 113, 20355 (2009)]. It was found that the toluene molecule is almost perpendicular to the silica surface with a tilt angle of about $25^{\circ}$. Here, we have investigated the structural properties of toluene and heptane at oil-silica interfaces using molecular dynamics simulations for two different surfaces: the oxygen-bridging (hydrophobic) and hydroxyl-terminated (hydrophilic) surfaces of quartz (silica). Based on the density profile, it was found that both heptane and toluene oscillate on silica surfaces, with heptane showing more oscillation peaks. Furthermore, the toluene molecules of the first layer were found to have an asymmetric distribution of orientations, with more $\mathrm{CH}_{3}$ groups pointed away from the silica surface than towards the silica surface. These findings are generally consistent with previous experiments, and reveal enhanced molecular structures of liquids at oil-silica interfaces. () 2012 American Institute of Physics. [http://dx.doi.org/10.1063/1.4742696]
\end{abstract}

\section{INTRODUCTION}

The experimental probe of molecules at interfaces is challenging because of the buried nature and relatively small size, typically only a few molecular diameters wide. ${ }^{1-21}$ It has been shown using x-ray scattering with the beam line of the synchrotron light source ${ }^{2,3}$ that the liquid molecules pack in the vicinity of a solid surface such that the density profile of the liquid phase oscillates around the bulk value with a periodicity of about one molecular diameter. In the presence of a nearby second solid surface, the liquid is squeezed out and the oscillation effect for the "confined" liquid increases. The oscillated solvation forces of confined systems have been proven by using surface force apparatus. ${ }^{4-10}$ Moving away from the solid surface, the solvation force oscillation shows a damped feature up to 2-10 molecular layers, depending on the shape, size, and internal rigidity of the molecules, as well as the water activity and temperature. ${ }^{4-10}$ It has been found in the case of the confined liquid that six minima (oscillations) in the solvation forces are observed for alkanes, while there are only four minima for toluene. ${ }^{7,8}$ However, no detailed structural properties are available.

Sum frequency generation (SFG) vibrational spectroscopy has grown to be one of the most versatile and powerful analytical tool for investigating liquid-solid, liquid-liquid, and liquid-vapor interfaces. ${ }^{11-23}$ Because it is highly surfacespecific and applicable to all the interfaces accessible by light, SFG spectroscopy allows us to identify molecular species at interfaces by detecting molecular vibrational modes, and the orientation of the molecules by detecting the polarization dependence of the SFG signals. ${ }^{13-15,18}$ Toluene and heptane are of particular importance because they are the most commonly

\footnotetext{
a) Authors to whom correspondence should be addressed. Electronic addresses: y_liang@earth.kumst.kyoto-u.ac.jp and matsuoka@earth. kumst.kyoto-u.ac.jp.
}

used solvents for both industrial and scientific applications, and their binary mixture is often chosen to simulate the aromatic/aliphatic content of crude oil. ${ }^{24}$ The interfacial structure of toluene and heptane at oil-silica interfaces has been studied by SFG spectroscopy. ${ }^{14}$ However, a quantitative interpretation of the reported spectra is not straightforward. For example, it is not clear how the oscillating nature ${ }^{4-10}$ of liquid molecules in the vicinity of a solid surface is related to the measured SFG spectra.

In the petroleum industry, the structure of liquidrock interfaces needs to be well understood to optimize oil production. ${ }^{25}$ Sandstone reservoir minerals are usually represented by crystalline quartz and clay minerals. It is well-known that quartz, like the other inorganic oxides, owes its inherent hydrophilicity to surface hydroxyl groups. ${ }^{19,26,27}$ Different pretreatments have been employed in the measurements, ${ }^{14}$ although it is not fully understood how such treatments could potentially affect the silica surface, i.e., the ratio of surface hydroxyl groups and siloxane bridges. Therefore, it is of particular interest to examine how the purely oxygen bridging (hydrophobic) and fully hydroxylterminated (hydrophilic) silica surfaces could systematically reproduce the different interfacial structural properties and adsorption patterns of toluene and heptane.

Molecular dynamics and Monte Carlo simulations have developed rapidly and offer a molecular-level understanding of packing structures, adsorption, and other details of liquidsolid, liquid-liquid, and liquid-vapor interfaces ${ }^{28-47}$ that cannot easily be achieved solely through experiments. Most recently, the combination of simulations and SFG experiments have provided a detailed interfacial structure of the acetonitrile-silica interface and successfully reconciled the conflicting interpretations of a wide range of previous spectroscopic studies. ${ }^{22,47}$ Here, we have undertaken molecular dynamics simulations of heptane and toluene at the oil-silica interface. First, we studied how heptane and toluene behave 


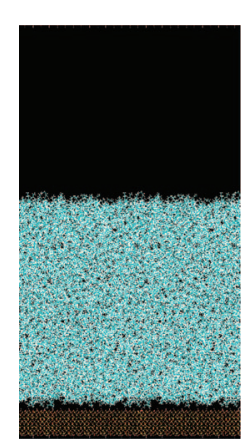

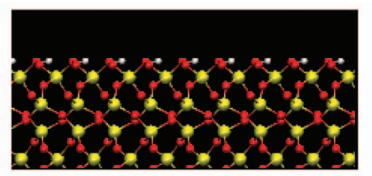

(b)

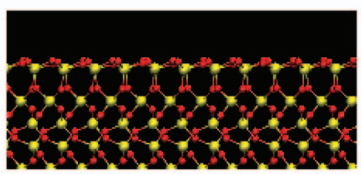

(c)

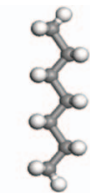

(d)

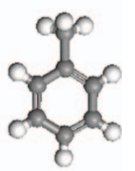

(e)
FIG. 1. Snapshots of heptane/toluene-quartz interface systems. (a) The liquid (in blue) is placed on top of the silica slab, which is either the hydrophilic (b), or hydrophobic (c) silica surface. Key: red = oxygen; yellow = silicon; and white $=$ hydrogen. The snapshot was repeated twice along the horizontal direction. The molecular structures of heptane and toluene are shown in (d) and (e). Note that the hydrophilic silica surface structure is terminated with germinal $\mathrm{Si}(\mathrm{OH})_{2}$ groups and the hydrophobic silica surface is terminated by siloxane $\mathrm{Si}-\mathrm{O}-\mathrm{Si}$ bridges.

at the oil-silica interface at the molecular scale in terms of the molecular oscillations and orientations. Second, we investigated how these orientations and oscillations will be reflected by SFG spectroscopy. And finally, we have explored the mechanisms of how to form the specific orientation from the interaction between the silica surface and the interfacial liquid molecules.

\section{COMPUTATIONAL METHODS}

\section{A. Quartz (silica) surface}

Each silica polymorph has a specific surface structure. For example, the fully hydroxylated $\alpha$-quartz (001) surface contains vicinal silanol, which has zigzag hydrogen-bonded networks with both short and long hydrogen bonds. ${ }^{48-50}$ The hydrophilic silica surface in this study was constructed by cleaving the bulk crystal of $\alpha$-quartz (space group $\mathrm{P} 3_{1} 21$ ) along the [001] direction. The fresh silica surface, which is terminated by $\mathrm{O}$ atoms, was saturated by manually adding $\mathrm{H}$ atoms to create the hydrophilic surface. We performed the first principles calculations using density functional theory with the Vienna $a b$ initio simulation package, ${ }^{51}$ similar to what has been described for previous simulations. ${ }^{49}$ Our final hydrophilic surface structure was in good agreement with the previous study, as shown in Fig. 1. For the hydrophobic silica surface, we used the reconstructed structure reported by Rignanese et $a .^{52}$ The surface has three- and six-membered rings after reconstruction of the newly cleaved surface with siloxane bridges. The surface areas $(\mathrm{x}, \mathrm{y})$ of the hydrophilic and hydrophobic silica surfaces were $5.9172 \times 6.8400 \mathrm{~nm}^{2}$ and $5.8920 \times 6.8032 \mathrm{~nm}^{2}$, respectively, with a thickness $(z)$ of about $2 \mathrm{~nm}$ for both silica slabs. The surface density of hydroxyl groups on the hydrophilic silica is $9.5 \mathrm{OH} / \mathrm{nm}^{2}$, while there are no hydroxyl groups present on the hydrophobic surface $\left(0 \mathrm{OH} / \mathrm{nm}^{2}\right)$.

\section{B. Details of molecular dynamics simulations}

The GROMACS package was used to perform the molecular dynamics of binary liquid heptane-toluene mixtures on top of the silica surfaces. ${ }^{53}$ The hydrocarbon compounds (heptane and toluene) were modeled using the CHARMM27 force field. ${ }^{54}$ The CLAYFF force field was chosen for both the hydrophilic and hydrophobic silica slabs. ${ }^{55-57}$ It has been shown that the CLAYFF force field reproduces the first-principles molecular dynamics results very well for the local structure of the silica-water interface. ${ }^{57}$ The electrostatic interactions were treated by particle mesh Ewald summation ${ }^{58}$ and a cutoff of $12 \AA$ was used for the van der Waals interactions.

The pure liquid heptane and toluene were generated by compressing the vapor phase at a constant temperature of $298 \mathrm{~K}$ and a pressure of 1 bar for $3.0 \mathrm{~ns}$. After the liquid formed, the mixture of heptane and toluene was composed of three variations in mole fraction $(1: 3,1: 1$, and $3: 1)$. The equilibrated densities of the liquid phases were 861.5, 803.4, $761.8,725.6$, and $699.8 \mathrm{~kg} / \mathrm{m}^{3}$ for pure toluene, the three mixtures, and pure heptane, respectively. They are in good agreement with the corresponding experimental values of 865.9, $803.9,758.2,713.0$, and $683.9 \mathrm{~kg} / \mathrm{m}^{3} .{ }^{59}$ All liquids contained the same number of molecules (2064 molecules), which were placed on the hydrophilic and hydrophobic silica surface. As shown in Fig. 1, the interface system consisted of three phases, i.e., solid (silica slab), liquid (heptane and toluene), and vapor (or vacuum), with the vertical size (along $z$ ) of the box being $25 \mathrm{~nm}$ for all calculations. The simulations were performed at a constant temperature of $300 \mathrm{~K}$ using the NoseHoover thermostat. ${ }^{60}$ The total simulation times were $3.0 \mathrm{~ns}$ for the pure liquids and $4.0 \mathrm{~ns}$ for the liquid mixture. The structural properties of the interface system were analyzed using the last $2.0 \mathrm{~ns}$ for every $1.0 \mathrm{ps}$ of the trajectory. The snapshots were prepared using Visual Molecular Dynamics software. ${ }^{61}$

\section{Order parameter}

For the orientational preference of the heptane and toluene molecules near the interface, we calculated the order parameter $^{43}$

$$
\mathrm{S}(\mathrm{z})=\frac{3}{2}\left\langle\cos ^{2} \theta(z)\right\rangle-\frac{1}{2},
$$

where $\theta$ is the angle between the molecular axis and the interface normal ( $z$ axis) and $\langle\cdots\rangle$ denotes the averaging over time and number of molecules. $z$ is the $z$-component of the Cartesian coordinate of the center of the mass for any given molecule and the vapor side of silica slab (i.e., the bottom in Fig. 1(a)) is chosen as the origin. $S(z)$ ranges from $-1 / 2$ to 1 , where $-1 / 2$ indicates perfectly perpendicular between two axes and 1 indicates parallel. It is zero if there is no preferred orientation. The molecular axis of heptane is defined as the vector from the head carbon site $\mathrm{C}_{1}$ to the tail carbon site $\mathrm{C}_{7}$, where the head and tail are labeled randomly. The molecular axis of toluene is defined by two different methods: (i) the vector from the para-carbon of the phenyl ring to the carbon of the methyl group; and (ii) the normal direction of the phenyl ring plane. Only by a combination of the two different vectors is it possible to explicitly describe the orientation of toluene on the top of the silica surface. 


\section{Angular dependent radial distribution}

Similar to our previous study on the benzene-water interface and benzene aqueous solutions, ${ }^{44}$ we calculated the angular dependent radial distribution function of $\mathrm{Si}-\mathrm{OH}$ from the hydrophilic silica surface and the center of the phenyl ring of toluene as follows:

$$
\begin{aligned}
g_{A B}(r, \Theta)= & \frac{\left\langle\rho_{B}(r, \Theta)\right\rangle}{\left\langle\rho_{B}\right\rangle_{\text {local }, \Theta}} \\
= & \frac{1}{\left\langle\rho_{B}\right\rangle_{\text {local }, \Theta}} \frac{1}{N_{A}} \sum_{i \in A}^{N_{A}} \\
& \times \sum_{j \in B}^{N_{B}} \frac{\delta\left(r_{i j}-r\right) \delta\left(\Theta_{i j}-\Theta\right)}{2 \pi r^{2} \sin \Theta},
\end{aligned}
$$

where $\left\langle\rho_{\mathrm{B}}(\mathrm{r}, \Theta)\right\rangle$ is the particle density of atom $B$ at a distance $r$ and angle $\Theta$ around atom $A . \delta$ is the delta function. The distribution function described here has been shown to successfully describe the interplay between the hydrogen site of water and the center of the benzene ring via weak hydrogen bonding. ${ }^{44}$ Therefore, it is interesting to verify whether a weak hydrogen bond is formed between the $\mathrm{Si}-\mathrm{OH}$ group of silica and the phenyl ring of toluene.

\section{RESULTS AND DISCUSSION}

\section{A. Density profiles}

Segmental density profiles for pure heptane, pure toluene, and their binary mixture on top of the hydrophilic and hydrophobic silica surfaces are shown in Fig. 2. The oscillation pattern was observed up to $\sim 3-3.5 \mathrm{~nm}$ of $z$ for all compositions. The highest intensity occurs in the nearest liquid-solid interface, followed by several peaks with lower intensity until the bulk phase. The first peak in the density profile indicates a strong anisotropic interaction between liquid molecules and the silica slab. As shown in Figs. 2(a) and 2(f), heptane has four peaks, similar to the oscillation pattern of $n$-octane between hydroxylated $\alpha-\mathrm{Al}_{2} \mathrm{O}_{3}(0001)$ surfaces at $300 \mathrm{~K}$, which is an analog of the hydrophilic silica surface. ${ }^{38}$ On the other hand, toluene shows only two clear peaks in the density profile (Figs. 2(e) and 2(j)). These results are in good agreement with the surface force experiment, where linear molecules like heptane and octane usually show more oscillation peaks than planar molecules like toluene. ${ }^{7,8}$

In the case of the mixture, we assumed that toluene acts as an impurity of heptane. On the hydrophilic silica surface, we observed that the oscillation pattern of heptane varied and that the first peak decreased significantly compared with pure heptane, even with a small amount of toluene. At high concentrations of toluene, the first peak of heptane was not observed. This means that more toluene than heptane is adsorbed on the hydrophilic silica surface. Different results were observed for the hydrophobic silica surface. As shown in Fig. 2(i), the first peak of heptane can still be observed for the mixture, although the intensity decreases with increasing toluene concentration. The different response of heptane upon the addition of toluene for the hydrophilic and hydrophobic silica surfaces can be attributed to the different polarities of the two silica surfaces. The polarity of silica surface increases with increasing density of silanol groups. ${ }^{42}$ The surface with a
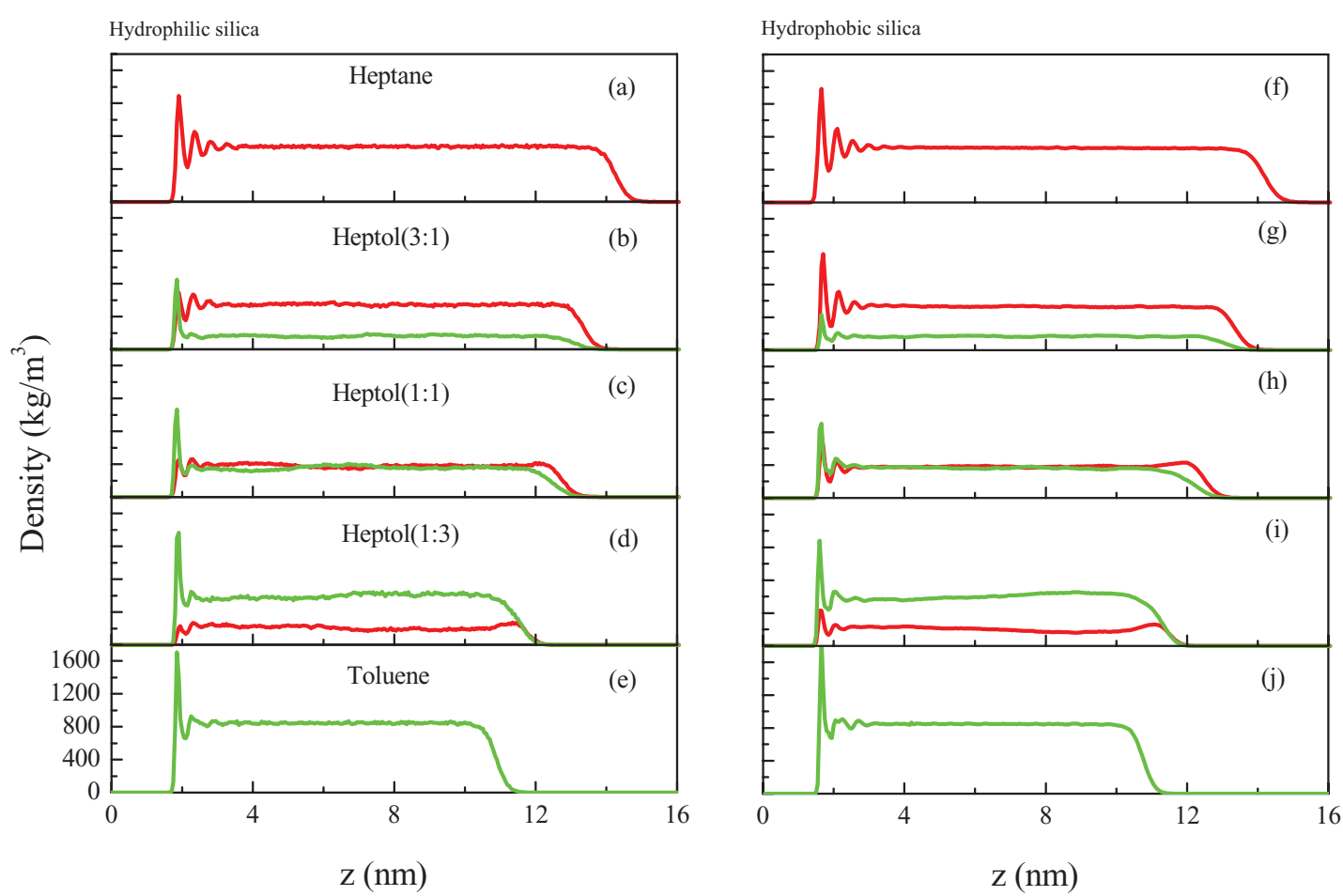

FIG. 2. Segmental density profiles along the normal direction of the interface ( $z$ ) of heptane (red), toluene (green), and their mixture on top of the hydrophilic ((a)-(e)) and hydrophobic surface ((f)-(j)). Heptol refers the heptane-toluene mixture. Note that pure heptanes, (a) and (f), have more oscillations than pure toluene, (e) and (j). 
(a) Heptane + Hydrophilic

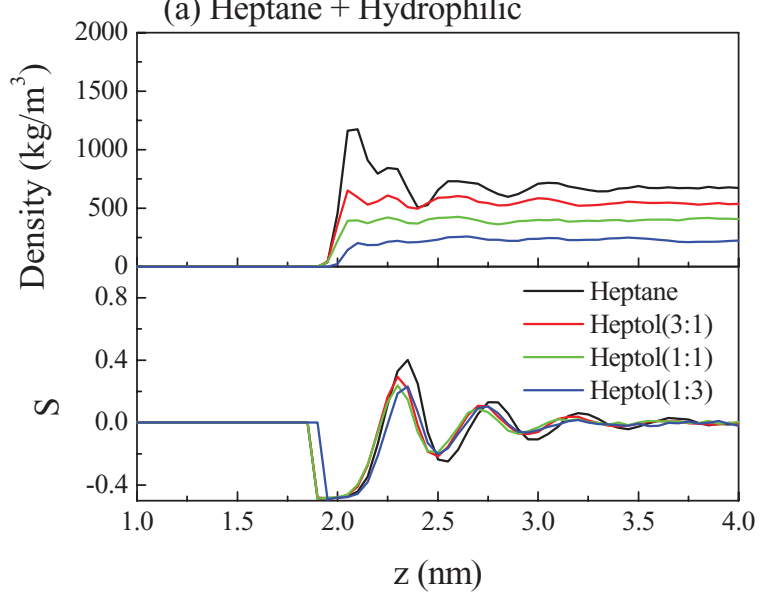

(b) Heptane + Hydrophobic

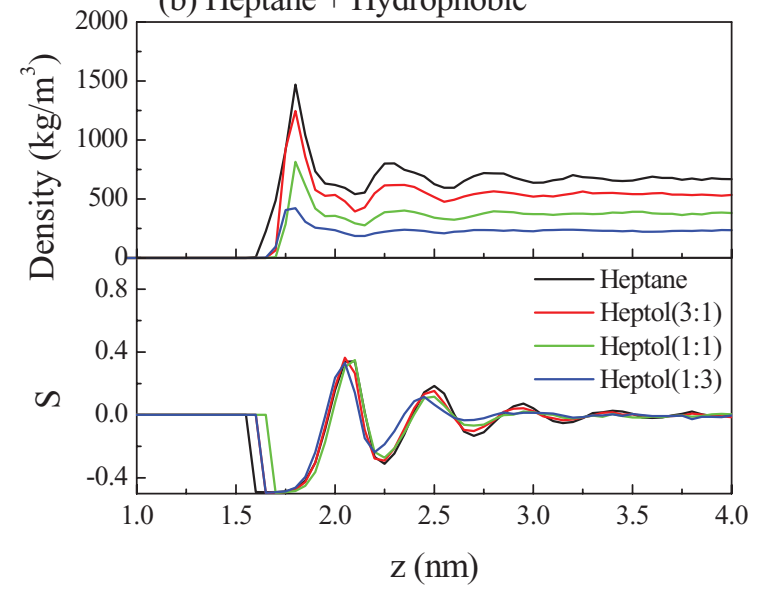

(c) Toluene + Hydrophilic

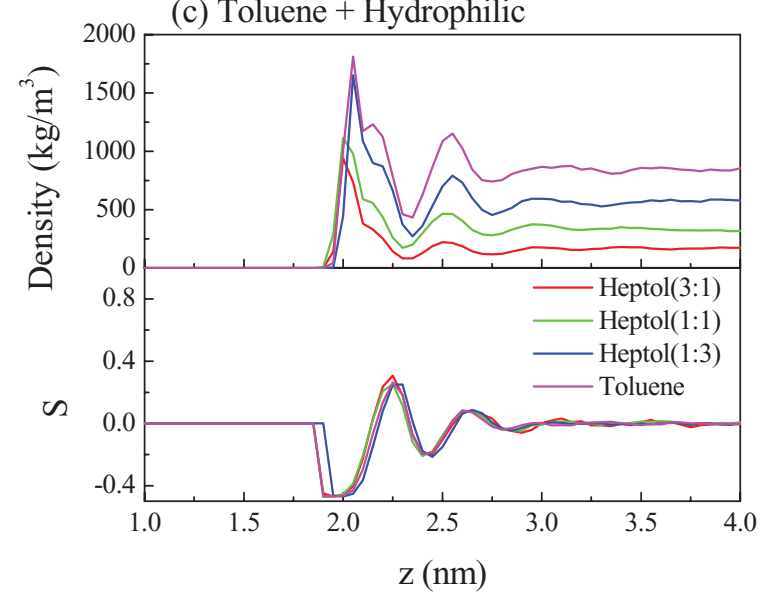

(d) Toluene + Hydrophobic

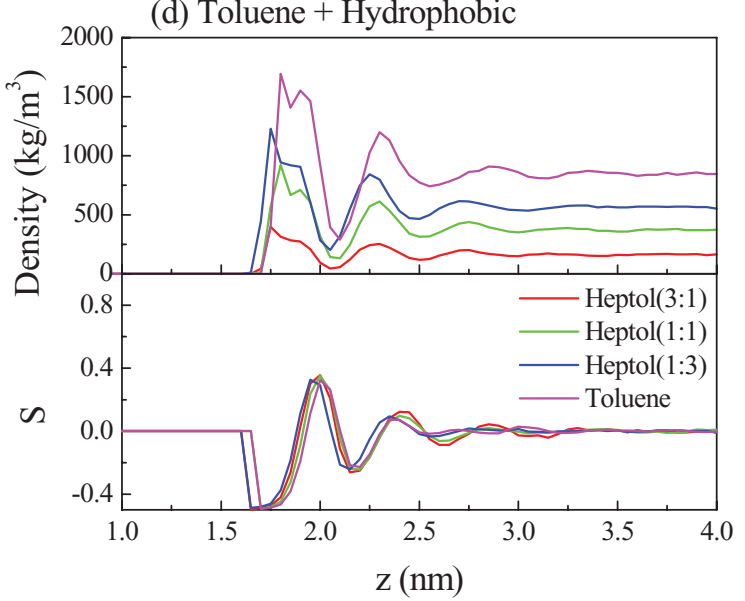

FIG. 3. Preferred orientations of heptane and toluene on hydrophilic ((a) and (c)) and hydrophobic ((b) and (d)) silica surface as reflected by the order parameter defined in Eq. (1). The center-of-the-mass density profiles, corresponding to the orientation of molecules, are shown in the top parts of the respective panels.

high polarity is attractive to aromatics, like toluene, and other polar compound. Similar behavior was observed for benzene on top of silica surfaces with various polarities. The surface with a high density of silanol groups $\left(7-8 \mathrm{OH} / \mathrm{nm}^{2}\right)$ was found to attract more benzene molecules at the interface than for a lower density surface $\left(2 \mathrm{OH} / \mathrm{nm}^{2}\right)$, as reported in a recent Monte Carlo simulation. ${ }^{42}$

\section{B. Orientation profiles}

The strong interaction of the solid and liquid at the interface results in liquid molecules adsorbing with certain orientations with respect to the solid surface. The liquid molecules, which usually have random orientations in the bulk liquid, become well-ordered at the interface. As you move away from the solid surface, the interactions become weaker and slowly disappear at the bulk liquid phase. The orientation of molecules near the surface is calculated by the order parameter (see Sec. II C for the definition). The normal direction of the surface $(z)$ has been chosen as the surface axis, while the axis of heptane and toluene are along the chain from head to tail carbon and from para-carbon to the methyl group, respectively. Therefore, if heptane or toluene has an orientation parallel to surface, the $S$ value will equal $-1 / 2$. As shown in Fig. 3, both heptane and toluene molecules lie nearly parallel to the hydrophilic and hydrophobic silica surfaces in the first layer (the first minimum in Fig. 3). There are no clear mixture effects in the orientation profile. The fact that heptane lies nearly parallel to the silica surface is similar to other linear chain alkanes, ${ }^{34}$ and also in excellent agreement with the SFG measurements. ${ }^{14}$ In the case of toluene, however, another possibility is that the normal of the phenyl ring may be parallel to the silica surface, i.e., the phenyl plane is perpendicular to the silica surface. Thus, we have defined another vector using the normal of the phenyl ring, where "up" and "down" are randomly chosen and the reference direction is still the normal direction of the silica surface. We have found that the $S$ of the first layer is almost 1. This indicates that the phenyl ring does indeed lie parallel to the silica surface. This finding is in contrast to the SFG measurement, where the toluene molecules of the first layer adopt an upright orientation with a tilt angle of $\sim 25^{\circ}$ with respect to the normal to the surface.

To attempt to explain the difference, we have investigated the interface structure in more detail. As the orientation parameter (Fig. 3) is based only on the molecule while the segmental density profile (Fig. 2) is based on the individual atoms, it would be interesting to introduce the density profile on the basis of the center of mass as well. As shown in Fig. 3, a similar oscillation was also observed in the center of mass density profiles with slightly different features. 


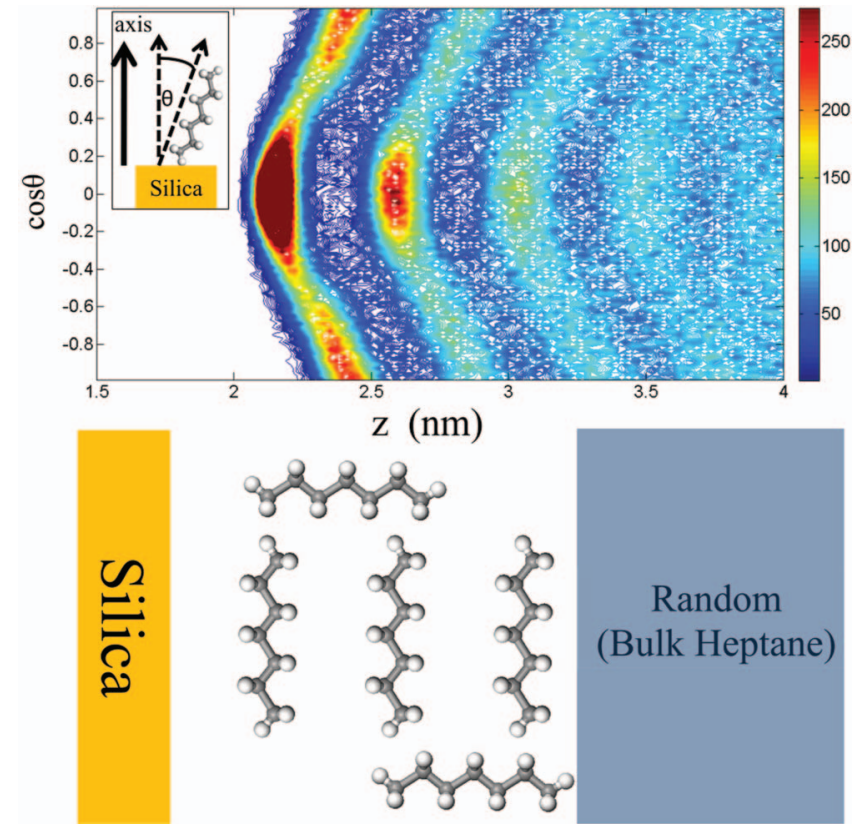

FIG. 4. Two-dimensional orientation profile of heptane on the hydrophilic silica surface (top) and a schematic representation of the preferred orientation of heptane in the vicinity of the silica surface (bottom). Inset: the angle is defined on the basis of the molecule axis and the surface normal direction.

In particular, we observed the appearance of shoulder peaks and shifted maximum-minimum in the center of mass density profiles. Because we notice that the differences in the minimum and maximum on the orientation profile originated from the same peak (i.e., a main peak with a shoulder peak) in the center of mass density profile, we have named the first minimum and maximum as first and second "sub-layers" of the first layer, respectively.

To obtain more detailed information about the orientations of the first and second sub-layers, we have analyzed the two-dimensional orientation profile, the distribution as a function of the angle $(\theta)$ between the molecule axis and the surface normal direction, and the $z$-component of the Cartesian coordinate of the center of the mass for that molecule (z). We took the case of pure heptane and toluene on top of the hydrophilic surface as illustrated in Figs. 4 and 5. The layering of heptane and toluene molecules can be clearly seen, where two clear patterns are repeated about every $0.5 \mathrm{~nm}$ in the density profiles. We confirmed that heptane forms more layers than toluene, as shown previously in the density profiles. The first sub-layer of heptane was dominated by the molecules with $\cos \theta$ from -0.4 to 0.4 , which means that heptane mostly lies on top of the hydrophilic silica surface, as shown in the bottom panel of Fig. 4. Furthermore, heptane in the second sublayer was found to be almost perpendicular to the silica surface with $-0.8<\cos \theta<-1.0$ or $0.8<\cos \theta<1.0$.

The second layer shows a similar trend before reaching bulk heptane at $z=3.5 \mathrm{~nm}$. As shown in Fig. 5, the first sub-layer of toluene is dominated by molecules lying parallel to the surface, similar to the case of heptane. Surprisingly, we observe a clear asymmetric orientation pattern of toluene molecules, i.e., positive $\cos \theta$ was found to be more frequent than negative $\cos \theta$ (as shown in Fig. 5). As $\theta$ is defined on the
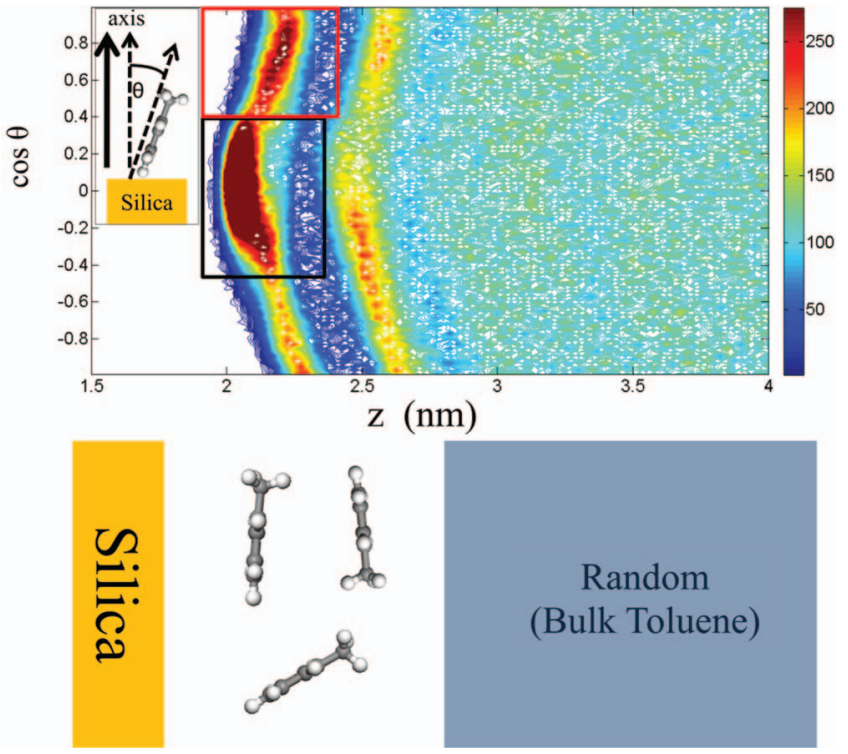

FIG. 5. Two-dimensional orientation profile of toluene on the hydrophilic silica surface (top) and a schematic diagram of the preferred orientations of toluene in the vicinity of the silica surface (bottom). Inset: the angle is defined on the basis of the molecule axis (from the para-carbon of the phenyl ring to the carbon of the methyl group) and the surface normal direction. Note that the region in the black rectangular $(|\cos \theta| \leq 0.4$ and $1.9 \leq z \leq 2.35)$ indicates the parallel geometry, i.e., the first sub-layer. The region in the red rectangular $(\cos \theta>0.4$ and $1.9 \leq z \leq 2.4)$ indicates the upright geometry with the $\mathrm{CH}_{3}$ group pointed away from the silica surface. The remainder of the first layer is the upright geometry with $\mathrm{CH}_{3}$ groups pointing towards the silica surface.

basis of the axis from the para-carbon of the phenyl ring to the carbon of the methyl group, this clearly indicates that the toluene molecules of the second sub-layer adopts an upright geometry with the methyl group pointing away from the silica surface. Furthermore, it is found that toluene has a preferred orientation with respect to the surface and shows a maximum of $0.8-0.9$ for $\cos \theta$, i.e., with a tilt angle of $25^{\circ}-35^{\circ}$, at the first peak position, which is $2.35-2.4 \mathrm{~nm}$ of the order parameter.

Figures 6(a) and 6(b) show the percentages of the upright and parallel orientations of toluene at different concentrations in the first layer. In the case of the pure toluene, we found that about $60 \%$ of the toluene molecules are parallel and about $40 \%$ are upright with respect to the silica surface. This ratio is almost constant on the hydrophobic silica surface with increasing heptane concentration (Fig. 6(b)). However, it increases to $\sim 70 \%$ with a heptane concentration equal to $75 \%$ mole fraction on the hydrophilic silica surface (Fig. 6(a)). As discussed below, this might be due to the specific weak hydrogen bonding between the phenyl ring of toluene and $\mathrm{Si}-\mathrm{OH}$ of the hydrophilic silica surface. The percentages of toluene molecules with $\mathrm{CH}_{3}$ groups pointed away from the silica surface are $55 \%-60 \%$ for the four different concentrations (Fig. 7(a)) on the hydrophilic silica surface and $52 \%-60 \%$ on the hydrophobic surface (Fig. 7(b)).

In summary, a schematic orientation at different (sub-) layers can be drawn as in Fig. 5 (bottom). The toluene molecules in the first sub-layer lie parallel to the silica surface, whereas in the second sub-layer they lie almost perpendicular to the silica surface with a tilt angle of $25^{\circ}-35^{\circ}$. We 

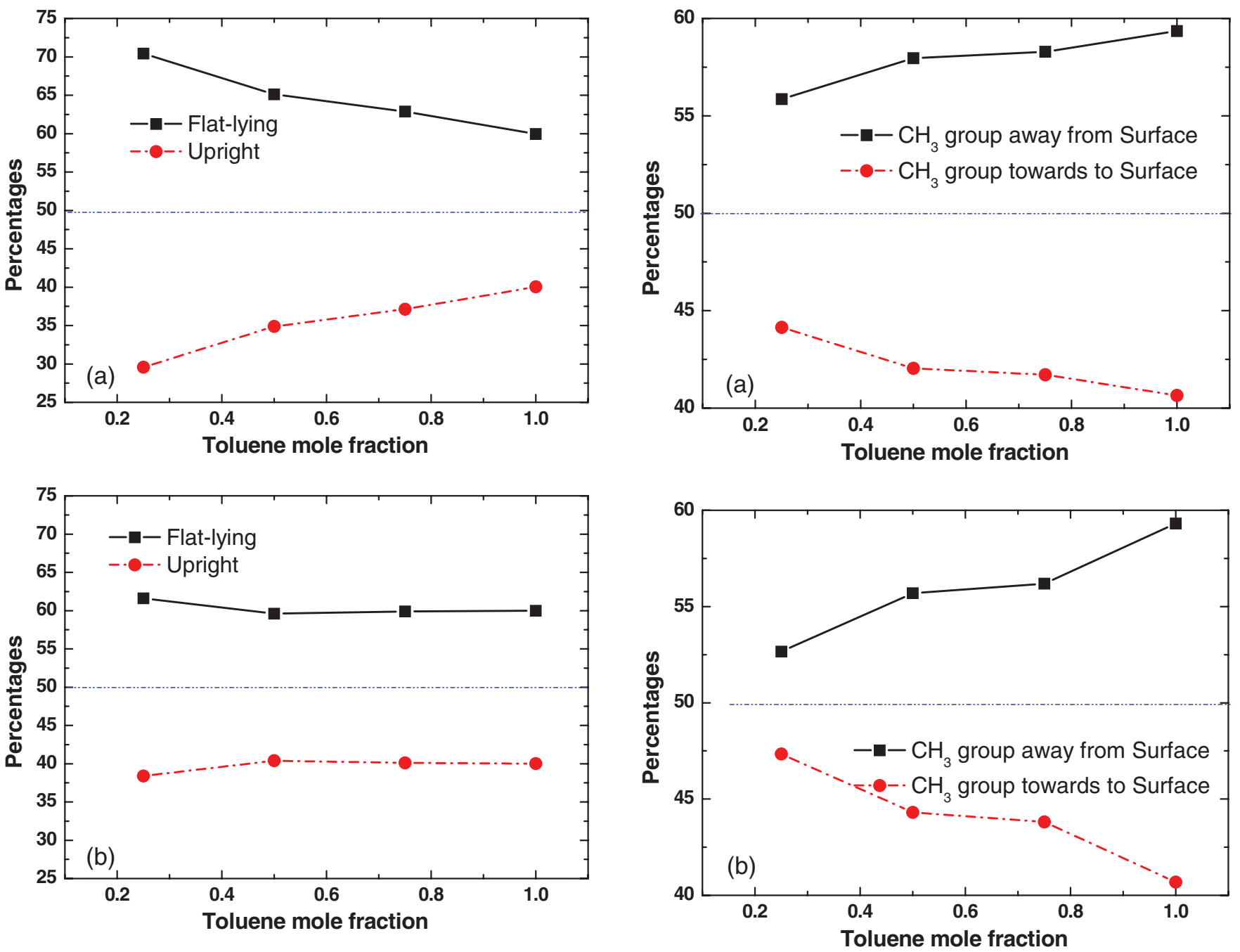

FIG. 6. Percentages of toluene molecules with parallel and upright geometries at different concentrations. (a) Hydrophilic and (b) hydrophobic surfaces.

suspect that the parallel toluene molecules might not be detectable by the SFG experiments, since the asymmetric peak of the $\mathrm{CH}_{3}$ group of the toluene molecule is not well-defined and the symmetric peak of the $\mathrm{CH}_{3}$ group will be missing with this orientation. ${ }^{14}$

\section{Mechanisms: Interplay between silica surface and first-layer toluene molecules}

The parallel toluene and heptane molecules on either silica surface could be due to the constraining effect of the solid wall. However, the fact that the ratio of the upright geometry molecules and the parallel molecules varies for the two different surfaces, i.e., there are more parallel toluene molecules on the hydrophilic surface than on the hydrophobic surface after adding heptane (Figs. 6(a) and 6(b)), indicates that there is a stronger interaction between the hydrophilic silica surface and the parallel molecules. Figures 8(a) and 8(d) show the angle dependent radial distribution functions of the $\mathrm{O}$ and $\mathrm{H}$ atom of the hydrophilic silica surface to the normal of the ring of toluene. In this figure, the maximum intensity for find-

FIG. 7. Percentages of toluene molecules with the $\mathrm{CH}_{3}$ group pointing away from and towards the silica surface at different concentrations. (a) Hydrophilic and (b) hydrophobic surfaces.

ing the $\mathrm{O}$ atom of the hydrophilic silica interface with an angle less than $20^{\circ}$ to the normal of the phenyl ring of toluene is $\sim 0.3 \mathrm{~nm}$. The highest intensity for the $\mathrm{H}$ atom of the hydrophilic quartz interface is located at $\sim 0.2 \mathrm{~nm}$, and at an angle less than $20^{\circ}$ from the normal of phenyl ring. Considering the two orientations of toluene in the first layer, we have investigated the first and second sub-layer regions, and defined a rectangular block of the certain position $(z)$ and orientation $(\cos \theta)$ ranges, as labeled in Fig. 5. It shows that the maximum intensity for finding $\mathrm{O}$ and $\mathrm{H}$ atom of the hydrophilic silica from normal of the ring of toluene corresponds to the molecules in the first sub-layer only (Figs. 8(a)-8(f)). This indicates that the toluene molecules of the second sub-layer adopt an upright geometry with more $\mathrm{CH}_{3}$ groups pointed away from the silica surface, which is due to the interaction between these molecules and the molecules lying parallel to the surface, presumably by forming a T-shaped structure with the molecule from the first sub-layer. ${ }^{62}$ Finally, there is a slightly higher proportion of $\mathrm{CH}_{3}$ groups pointing away from the silica surface on the hydrophilic silica surface than on the hydrophobic surface at the same heptane concentration 


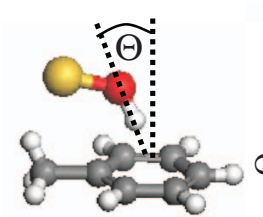

Total

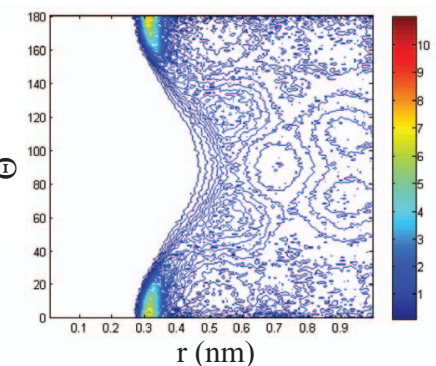

(a)

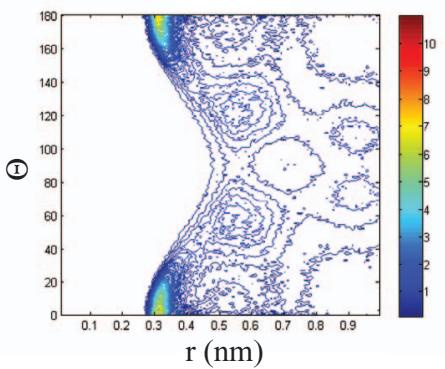

(b)

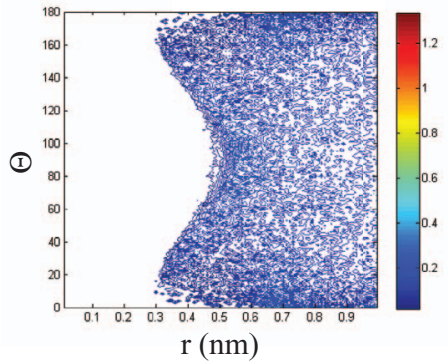

(c)

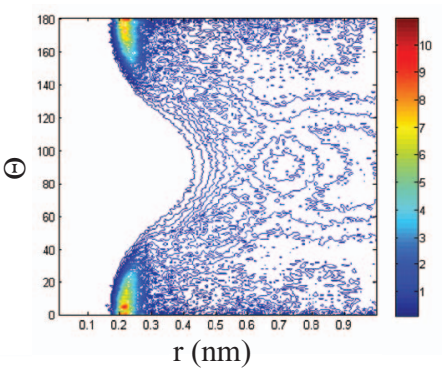

(d)

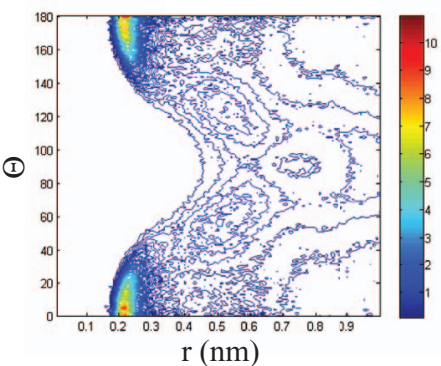

(e)

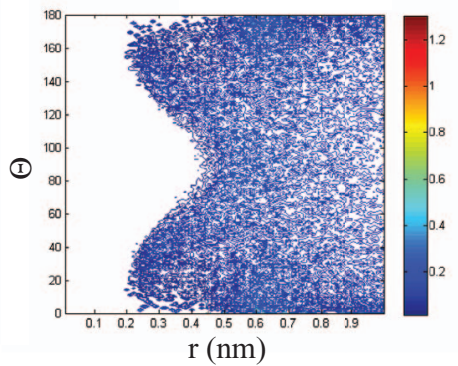

(f)

FIG. 8. Angular dependent radial distribution functions from the center of the phenyl ring of toluene to the oxygen ((a)-(c)) and hydrogen ((d)-(f)) atoms of $\mathrm{Si}-\mathrm{OH}$ on the hydrophilic silica surface. (a) and (d) are for the total system, (b) and (e) consider only the toluene molecules with parallel geometries, and (c) and (f) are those with an upright geometry.

(Figs. 7(a) and 7(b)), which can be attributed to the larger proportion of toluene molecules lying parallel to the hydrophilic surface, as mentioned above.

\section{CONCLUSIONS}

Our simulations have shown that heptane lies mostly parallel to the surface in the first layer. This result is in excellent agreement with recent SFG spectrum measurements, where all of the n-alkanes $(n=5,7$, and 14) were found to adopt the same orientation on the silica surface. The toluene molecules in the second sub-layer adopt an upright geometry with a tilted angle of $\sim 25^{\circ}$ with respect to the surface normal, which has also been found with recent SFG experiments. The presence of the methyl group in the toluene molecule leads to an asymmetric pattern of orientations with the methyl group pointing away from both the hydrophobic and hydrophilic silica surfaces. The knowledge obtained from our simulations can be used to aid in the understanding of the interfacial structure obtained by SFG spectroscopy.

\section{ACKNOWLEDGMENTS}

The authors acknowledge the financial support of the Japanese Society for the Promotion of Science (JSPS) through a Grant-in-Aid for Scientific Research A (No. 24246148), JOGMEC, JST/JICA-SATREPS, and JAPEX. We also wish to thank Koichi Takamura, Caetano R. Miranda, Yasuhiro Fukunaka, and Akira Ueda for valuable discussions. M.L. is supported by a Japanese Government Scholarship from the Ministry of Education, Culture, Sports, Science, and Technology. M.K. is grateful for a JSPS Research Fellowship through KAKENHI 226752.

${ }^{1}$ H.-J. Butt, K. Graf, and M. Kappl, Physics and Chemistry of Interfaces, 2nd ed. (Wiley-VCH, Weinheim, 2006).

${ }^{2}$ A. K. Doerr, M. Tolan, T. Seydel, and W. Press, Physica B 248, 263 (1998).

${ }^{3}$ C.-J. Yu, A. G. Richter, J. Kmetko, A. Datta, and P. Dutta, Europhys. Lett. 50, 487 (2000).

${ }^{4}$ R. G. Horn and J. N. Israelachvili, J. Chem. Phys. 75, 1400 (1981).

${ }^{5}$ H. K. Christenson, J. Chem. Phys. 78, 6906 (1983).

${ }^{6}$ H. K. Christenson and R. G. Horn, Chem. Phys. Lett. 98, 45 (1983).

${ }^{7}$ H. K. Christenson, D. W. R. Gruen, R. G. Horn, and J. N. Israelachvili, J. Chem. Phys. 87, 1834 (1987). 
${ }^{8}$ J. Klein and E. Kumacheva, J. Chem. Phys. 108, 6996 (1998). Detailed solvation force as a function of gap distance for toluene was not reported in this paper. However, it was reported that the sharp liquid-to-solid phase transition on increasing confinement between films of the thickness corresponds to four layers for toluene and six or seven layers for cyclohexane.

${ }^{9}$ M. L. Gee, P. M. McGuiggan, and J. N. Israelachvili, J. Chem. Phys. 93, 1895 (1990)

${ }^{10}$ H. K. Christenson, Chem. Phys. Lett. 118, 455 (1985).

${ }^{11}$ J. H. Hunt, P. Guyot-Sionnest, and Y. R. Shen, Chem. Phys. Lett. 133, 189 (1987).

${ }^{12}$ X. D. Zhu, H. Suhr, and Y. R. Shen, Phys. Rev. B 35, 3047 (1987).

${ }^{13}$ X. Zhuang, P. B. Miranda, D. Kim, and Y. R. Shen, Phys. Rev. B 59, 12632 (1999).

${ }^{14}$ Z. Yang, Q. Li, R. Hua, M. R. Gray, and K. C. Chou, J. Phys. Chem. C 113, 20355 (2009).

${ }^{15}$ M. R. Brindza, F. Ding, J. T. Foukas, and R. A. Walker, J. Chem. Phys. 132, 114701 (2010)

${ }^{16}$ Y. R. Shen, Nature (London) 337, 519 (1989).

${ }^{17}$ P. B. Miranda and Y. R. Shen, J. Phys. Chem. B 103, 3292 (1999).

${ }^{18}$ L. Zhang, W. Liu, Y. R. Shen, and D. G. Gahill, J. Phys. Chem. C 111, 2069 (2007).

${ }^{19}$ W.-T. Liu and Y. R. Shen, Phys. Rev. Lett. 101, 016101 (2008).

${ }^{20}$ O. Esenturk and R. A. Walker, J. Chem. Phys. 125, 174701 (2006).

${ }^{21}$ E. L. Hommel and H. C. Allen, Analyst 128, 750 (2003).

${ }^{22}$ F. Ding, Z. Hu, Q. Zhong, K. Manfred, R. R. Gattass, M. R. Brindza, J. T. Fourkas, R. A. Walker, and J. D. Weeks, J. Phys. Chem. C 114, 17651 (2010).

${ }^{23}$ A. M. Buchbinder, E. Weitz, and F. M. Geiger, J. Am. Chem. Soc. 132, 14661 (2010).

${ }^{24}$ A. Morita and T. Ishiyama, Phys. Chem. Chem. Phys. 10, 5801 (2008).

${ }^{25}$ A. Yeung, T. Dabros, and J. Maliyah, J. Colloid Interface Sci. 208, 241 (1998).

${ }^{26}$ L. W. Lake, Enhanced Oil Recovery (Prentice Hall, New Jersey, 1989).

${ }^{27}$ R. N. Lamb and D. N. Furlong, J. Chem. Soc. Faraday Trans. 1 78, 61 (1982).

${ }^{28}$ L. T. Zhuravlev, Colloids Surf., A 173, 1 (2000).

${ }^{29}$ F. F. Abraham, J. Chem. Phys. 68, 3713 (1978).

${ }^{30}$ M. Rao, B. J. Berne, J. K. Percus, and M. H. Kalos, J. Chem. Phys. 71, 3802 (1979)

${ }^{31}$ J. Gao, W. D. Luedtke, and U. Landman, Phys. Rev. Lett. 79, 705 (1997)

${ }^{32}$ L. G. Camara and F. Bresme, J. Chem. Phys. 120, 11355 (2004)

${ }^{33}$ D. K. Hore, D. S. Walker, and G. L. Richmond, J. Am. Chem. Soc. 129, 752 (2007).
${ }^{34}$ J. C. Wang and K. A. Fichthorn, J. Chem. Phys. 108, 1653 (1997).

${ }^{35}$ J. Gao, W. D. Luedtke, and U. Landman, J. Chem. Phys. 106, 4309 (1997).

${ }^{36}$ J. Gao, W. D. Luedtke, and U. Landman, J. Phys. Chem. B 101, 4013 (1997)

${ }^{37}$ V. Kalyanasundaram, D. E. Spearot, and A. P. Malshe, Langmuir 25, 7553 (2009)

${ }^{38}$ R. Y. Jin, K. Song, and W. L. Hase, J. Phys. Chem. B 104, 2692 (2000).

${ }^{39}$ H. Docherty and P. T. Cummings, Soft Matter 6, 1640 (2010).

${ }^{40}$ H. Matsubara, F. Pichierri, and K. Kurihara, J. Chem. Phys. 134, 044536 (2011).

${ }^{41}$ T. K. Xia and U. Landman, Science 261, 1310 (1993).

${ }^{42}$ B. Coasne, C. Alba-Simionesco, F. Audonnet, G. Dosseh, and K. E. Gubbins, Langmuir 25, 10648 (2009).

${ }^{43}$ A. R. van Buuren, S.-J. Marrink, and H. J. C. Berendsen, J. Phys. Chem. 97, 9206 (1993).

${ }^{44}$ M. Kunieda, K. Nakaoka, Y. Liang, C. R. Miranda, A. Ueda, S. Takahashi, H. Okabe, and T. Matsuoka, J. Am. Chem. Soc. 132, 18281 (2010).

${ }^{45}$ J. G. Harris, J. Phys. Chem. 96, 5077 (1992).

${ }^{46}$ M. Kawamata and T. Yamamoto, J. Phys. Soc. Jpn. 66, 2350 (1997).

${ }^{47}$ Z. Hu and J. D. Weeks, J. Phys. Chem. C 114, 10202 (2010).

${ }^{48}$ T. P. M. Goumans, A. Wander, W. A. Brown, and C. R. A. Catlow, Phys. Chem. Chem. Phys. 9, 2146 (2007).

${ }^{49}$ J. Yang and E. G. Wang, Phys. Rev. B 73, 035406 (2006).

${ }^{50}$ V. V. Murashov, J. Phys. Chem. B 109, 4144 (2005).

${ }^{51}$ G. Kresse and G. Furthmuller, Phys. Rev. B 54, 11169 (1996).

${ }^{52}$ G. M. Rignanese, A. D. Vita, J. C. Charlier, X. Gonze, and R. Car, Phys. Rev. B 61, 13250 (2000)

${ }^{53}$ B. Hess, C. Kutzner, D. van der Spoel, and E. Lindahl, J. Chem. Theory Comput. 4, 435 (2008)

${ }^{54}$ J. B. Klauda, B. R. Brooks, A. D. Mackerrel, Jr., R. M. Venable, and R. W. Pastor, J. Phys. Chem. B 109, 5300 (2005).

${ }^{55}$ R. T. Cygan, J. J. Liang, and A. G. Kalinichev, J. Phys. Chem. B 108, 1255 (2004)

${ }^{56}$ A. A. Skelton, D. J. Wesolowski, and P. T. Cummings, Langmuir 27, 8700 (2011)

${ }^{57}$ A. A. Skelton, P. Fenter, J. D. Kubicki, D. J. Wesolowski, and P. T. Cummings, J. Phys. Chem. C 115, 2076 (2011).

${ }^{58}$ U. Essman, L. Perera, M. L. Berkowitz, T. Darden, H. Lee, and L. G. Pedersen, J. Chem. Phys. 103, 8577 (1995).

${ }^{59}$ E. C. Bromiley and D. Quiggle, Ind. Eng. Chem. 25, 1136 (1933).

${ }^{60}$ S. Nose, Mol. Phys. 52, 255 (1984).

${ }^{61}$ W. Humphrey, A. Dalke, and K. Schulten, J. Mol. Graph. 14, 33 (1996).

${ }^{62}$ C. M. Baker and G. H. Grant, J. Chem. Theory. Comput. 3, 530 (2007). 